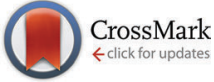

Cite this: J. Mater. Chem. C, 2016 , 4, 4763

Received 15th March 2016, Accepted 21st April 2016

DOI: $10.1039 / c 6 t c 01107 a$

www.rsc.org/MaterialsC

\section{Perovskite ferroelectrics and relaxor-ferroelectric solid solutions with large intrinsic electrocaloric response over broad temperature ranges}

\author{
H. Khassaf, ${ }^{a}$ J. V. Mantese, ${ }^{b}$ N. Bassiri-Gharb, ${ }^{c}$ Z. Kutnjak $^{d}$ and S. P. Alpay*a
}

\begin{abstract}
Electrocaloric materials have emerged as a viable technology for solid state heating/cooling and waste heat recovery applications. We determine here intrinsic electrocaloric (EC) entropy and temperature changes in perovskite ferroelectrics (FEs) using theoretical tools supported by experimentally measured heat capacities as a function of the applied electric field and temperature. A quantitative theoretical analysis of the thermal, pyroelectric and EC properties of representative ceramic FE systems is provided for $\mathrm{BaTiO}_{3}$ and $\mathrm{PbTiO}_{3}(\mathrm{PT})$ that display a weak and a strong first-order phase transformation to the $\mathrm{FE}$ state, and relaxor- $\mathrm{FE} x \mathrm{~Pb}\left(\mathrm{Mg}_{1 / 3} \mathrm{Nb}_{2 / 3}\right)$ $\mathrm{O}_{3}-(1-x) \mathrm{PbTiO}_{3}[x \mathrm{PMN}-(1-x) \mathrm{PT}]$ solid solutions. Our results indicate that the intrinsic adiabatic temperature changes in relaxor-FEs are substantial. Temperature variations (related to reduced entropy) as high as $14{ }^{\circ} \mathrm{C}$ can be achieved with applied fields on the order of $1 \mathrm{MV} \mathrm{cm}^{-1}$ at $T \sim 350{ }^{\circ} \mathrm{C}$ for (001) oriented 0.65PMN-0.35PT. Moreover, the EC response does not vary over a large temperature interval: $13 \pm 1{ }^{\circ} \mathrm{C}$ for $200<T<600{ }^{\circ} \mathrm{C}$. In PT, the adiabatic temperature change is approximately $28^{\circ} \mathrm{C}$ for an applied field of $1 \mathrm{MV} \mathrm{cm}^{-1}$, rivaling the best EC response observed in polymer FEs. This study provides the general methodology for theoretical analysis to both assess and guide the way in discovering newer high performance EC materials.
\end{abstract}

\section{Introduction}

Since the first observance nearly a decade ago of a giant electrocaloric (EC) effect in thin film ferroelectric $\mathrm{PbZr}_{0.95} \mathrm{Ti}_{0.05} \mathrm{O}_{3}$ by Mischenko et al. ${ }^{1}$ the field of ferroelectric (FE) based solid state electrothermal interconversion has seen rapid advancement, significantly improving both the magnitude of usable EC adiabatic temperature change $\Delta T_{\mathrm{EC}}$, and the available cooling capacity as defined by an enthalpy $(\Delta H)$ or entropy $(\Delta S)$ variation: $\Delta H=\Delta S \cdot \Delta T_{\mathrm{EC}} \cdot{ }^{2-5}$ Several materials systems have been proposed for use in electrothermal applications including FE/anti-FE ceramics in their single-crystal, bulk, and thin film forms, FE polymers/co-polymers/terpolymers, and liquid crystals. ${ }^{6-13}$ There are also reports showing that relatively wide temperature range EC properties can be achieved in composites of ceramic and polymer relaxor FEs. ${ }^{14}$ These efforts have primarily been led through experimentation, augmented by expert intuition. ${ }^{15}$ The

\footnotetext{
${ }^{a}$ Department of Materials Science and Engineering and Institute of Materials Science, University of Connecticut, Storrs, CT 06269, USA.

E-mail: pamir.alpay@uconn.edu

${ }^{b}$ United Technologies Research Center, East Hartford, Connecticut 06118, USA ${ }^{c}$ G.W. Woodruff School of Mechanical Engineering, and School of Materials Science and Engineering, Georgia Institute of Technology, Atlanta, Georgia 30332-0405, USA

${ }^{d}$ Jozef Stefan Institute and Jozef Stefan International Postgraduate School, 1000 Ljubljana, Slovenia
}

success of such an approach is demonstrated by the more than fourfold improvement in $\Delta T_{\mathrm{EC}}$ with a concomitant increase in $\Delta H$ of similar magnitude. ${ }^{4}$ Fundamental enhancements in material properties of this kind directly impact system level performance and costs, reducing the effects of parasitic losses and potentially yielding system level performance competitive with vapor compression systems. ${ }^{7}$

Many applications of FEs, from random memory (FRAMs) devices, ${ }^{16}$ to piezoelectric actuators,${ }^{17,18}$ and pyroelectric infrared detectors, ${ }^{19}$ have all benefitted (and often been directed) by multi-scale theoretical approaches including ab initio and beyond first principles methods, ${ }^{20,21}$ non-linear thermodynamics, and atomistic models. $^{22-24}$ Thus, it is compelling to employ similar methods as a means for understanding the material properties and discovering new materials systems for ECs. We present here a complete theoretical analysis of intrinsic adiabatic temperature changes in perovskite FEs using computational tools supported by carefully measured heat capacities ${ }^{25}$ as a function of the applied electric field $(E)$ and temperature $(T)$. Specifically, thermal, pyroelectric and EC properties of prototypical FEs $\mathrm{BaTiO}_{3}$ (BT) and $\mathrm{PbTiO}_{3}(\mathrm{PT})$, and relaxor-FEs $x \mathrm{~Pb}\left(\mathrm{Mg}_{1 / 3} \mathrm{Nb}_{2 / 3}\right) \mathrm{O}_{3}-(1-x) \mathrm{PbTiO}_{3}$ $[x \mathrm{PMN}-(1-x) \mathrm{PT}]$ compounds are calculated. Two specific compositions of PMN-PT are chosen as representatives of a relaxor and a relaxor-FE solid solution, 0.90PMN-0.10PT and $0.65 \mathrm{PMN}-0.35 \mathrm{PT}$, respectively. $0.90 \mathrm{PMN}-0.10 \mathrm{PT}$ is a relaxor composition that is far from the morphotropic phase boundary 
(MPB). 0.65PMN-0.35PT lies in close proximity of the MPB, where pseudo-rhombohedral, pseudo-monoclinic/orthorhombic, and pseudo-tetragonal phases can co-exist and phase transitions can be induced through applied electric and elastic fields. ${ }^{26}$ This system is of particular interest because it displays a strong orderdisorder and crystallographic phase transitions which couple with external stimuli. These particular characteristics are arguably at the core of the very large electromechanical response exhibited by $x \mathrm{PMN}-(1-x) \mathrm{PT}$ (with or without additional doping) which are used in high-strain actuators/transducers. ${ }^{27}$ The very large electromechanical response has been attributed to the inherently (intrinsic) large piezoelectric response that stems from ionic displacements, ${ }^{28}$ a large "extrinsic" contribution from highly adaptive nano- and mesoscale domain structures, ${ }^{29}$ and a strong contribution from electrostriction. ${ }^{30}$ A relatively large temperature change of $\sim 5{ }^{\circ} \mathrm{C}$ at an applied field difference of $\sim 900 \mathrm{kV} \mathrm{cm}^{-1}$ and $T=75{ }^{\circ} \mathrm{C}$ has been reported for this solid solution for $x=0.90 .^{26}$

Our analysis is based on the thermodynamic methodology that is described in detail in the next section. This study uncovers two significant findings. Firstly, the intrinsic adiabatic temperature changes in relaxor-FEs are substantial and relatively temperature insensitive. $\Delta T_{\mathrm{EC}}$ variations as high as $14{ }^{\circ} \mathrm{C}$ can be achieved with applied fields on the order of $1 \mathrm{MV} \mathrm{cm}^{-1}$ at $T \sim 350{ }^{\circ} \mathrm{C}$ for (001) oriented 0.65PMN-0.35PT. This EC response does not vary over a large temperature interval: $\Delta T_{\mathrm{EC}}=13 \pm 1{ }^{\circ} \mathrm{C}$ for $200<T<600{ }^{\circ} \mathrm{C}$. Secondly, our studies indicate that one can find perovskite FEs with exceedingly large intrinsic EC effects. $\Delta T_{\mathrm{EC}}$ is $\sim 28{ }^{\circ} \mathrm{C}$ in PT near its FE transition temperature for an applied field of $1 \mathrm{MV} \mathrm{cm}^{-1}$, rivaling the best EC response observed in polymer FEs. ${ }^{31,32}$ This is particularly important since only limited fundamental studies of the EC response exist in perovskite systems at high fields. ${ }^{33,34}$ The overarching purpose of this study is to provide a theoretical framework by which new candidate materials can be assessed for their EC properties and to guide experimental research in exploiting the full potential of existing materials.

\section{Methodology}

\subsection{Theoretical approach}

We consider a monodomain single crystal FE in an unconstrained, unstressed state. We analyze PT, BT, 0.90PMN-0.10PT, and
0.65PMN-0.35PT that undergo a cubic $(\mathrm{Pm} 3 \mathrm{~m})$ paraelectric (PE) to tetragonal $(P 4 \mathrm{~mm}) \mathrm{FE}$ transition at $T_{\mathrm{C}}$. The components of the polarization vector $\mathbf{P}$ for the $\mathrm{PE}$ and the $\mathrm{FE}$ phases are $P_{1}=P_{2}=P_{3}=0$ and $P_{1}=P_{2}=0, P_{3}=P \neq 0$, respectively. The volumetric (excess) free energy due to polarization is given by:

$$
G(T, E, P)=G_{0}+\alpha_{1} P^{2}+\alpha_{11} P^{4}+\alpha_{111} P^{6}-E P
$$

where $G_{0}$ is the free energy density of the (unpolarized) PE phase, and $\alpha_{1}, \alpha_{11}$, and $\alpha_{111}$ are dielectric stiffness coefficients. $\alpha_{1}$ is given by the Curie-Weiss law such that $\alpha_{0}=\left(T-T_{\mathrm{C}}\right) /$ $\left(2 \varepsilon_{0} C\right)^{-1}$ where $\varepsilon_{0}$ is the permittivity of space and $C$ is the CurieWeiss constant. In eqn (1), $\mathbf{E}=\left(0,0, E_{3}=E\right)$ is the applied electric field.

All thermodynamic properties and stiffness coefficients used in the computations are given in Table 1. For PMN-PT, we use Heitmann-Rossetti free energy potentials that were obtained using a combined phenomenological and topological analysis. ${ }^{35}$ The free energy potentials are supplemented by experimental "hard-mode" contributions to heat capacity determined experimentally [see description below and ref. 36]. The equilibrium polarization $P^{0}$ is then determined via solving the equation of state $\partial G / \partial P=0$.

The two most important factors in determining the adiabatic temperature change $\Delta T_{\mathrm{EC}}$ at a given $T$ with an applied electric field difference $\Delta E=\left(E_{\mathrm{b}}-E_{\mathrm{a}}\right)$ are: the pyroelectric coefficient of the material, $p$, and the heat capacity at constant $E, C_{E}^{\mathrm{XS}}$. The proper definitions of these quantities follow from the relevant Maxwell relations. At constant $E$, the excess entropy $S_{E}^{\mathrm{xS}}$, the excess specific heat $\Delta C_{E}$, and the pyroelectric coefficient $p$ follow from:

$$
\begin{gathered}
S_{E}^{\mathrm{XS}}(T, E)=-\left(\frac{\partial G^{0}}{\partial T}\right)_{E}, \\
\Delta C_{E}(T, E)=-T\left(\frac{\partial^{2} G^{0}}{\partial T^{2}}\right)_{E}, \\
p(T, E)=\left(\frac{\partial^{2} P^{0}}{\partial T}\right)_{E}=\left(\frac{\partial S}{\partial E}\right)_{T},
\end{gathered}
$$

$\Delta T_{\mathrm{EC}}$ as result of a change in the electric field strength $\Delta E=E_{b}-E_{a}$ can then be calculated via:

$$
\Delta T_{\mathrm{EC}}(T, E)=-T \int_{E_{a}}^{E_{b}} \frac{1}{C_{E}}\left(\frac{\partial P^{0}}{\partial T}\right)_{E} \mathrm{~d} E,
$$

Table 1 Property coefficients: property coefficients of BT, PT, 0.90PMN-0.10PT, and 0.65PMN-0.35PT. The data are compiled from ref. 18 and 23

\begin{tabular}{lllll}
\hline Parameter/material & BT & PT & 0.90 PMN-0.10PT & 0.65 PMN-0.35PT \\
\hline$T_{\mathrm{C}}\left({ }^{\circ} \mathrm{C}\right)$ & 121 & 475 & 38 & 159 \\
$\alpha_{1}\left(10^{5} \mathrm{~m} \mathrm{~F}^{-1}\right)$ & $3.76\left(T-T_{\mathrm{C}}\right)$ & $3.68\left(T-T_{\mathrm{C}}\right)$ & $1.81\left(T-T_{\mathrm{C}}\right)$ & $2.415\left(T-T_{\mathrm{C}}\right)$ \\
$\alpha_{11}\left(10^{6} \mathrm{~m}^{5} \mathrm{C}^{-2} \mathrm{~F}^{-1}\right)$ & $3.6 T-630$ & $-146-0.38 T$ & $61.6-0.038 T$ & $28.9-0.038 T$ \\
$\alpha_{111}\left(10^{9} \mathrm{~m}^{8} \mathrm{C}^{-4} \mathrm{~F}^{-1}\right)$ & 6.6 & 0.257 & 0.257 & 0.257 \\
$S_{11}\left(10^{12} \mathrm{~m}^{2} \mathrm{~N}^{-1}\right)$ & 8.3 & 7.7 & 0.018 & 0.31 \\
$S_{12}\left(10^{12} \mathrm{~m}^{2} \mathrm{~N}^{-1}\right)$ & -2.73 & -2.33 & -0.63 & -0.11 \\
$S_{44}\left(10^{12} \mathrm{~m}^{2} \mathrm{~N}^{-1}\right)$ & 9.22 & 8.77 & -0.56 & 0.245 \\
$Q_{11}\left(\mathrm{~m}^{4} \mathrm{C}^{-2}\right)$ & 0.11 & 0.09 & 0.084 & 0.084 \\
$Q_{12}\left(\mathrm{~m}^{4} \mathrm{C}^{-2}\right)$ & -0.034 & -0.025 & -0.025 & -0.025 \\
$Q_{44}\left(\mathrm{~m}^{4} \mathrm{C}^{-2}\right)$ & 0.029 & 0.039 & 0.035 & 0.035 \\
Hard mode $C_{E}\left(10^{6} \mathrm{~J} \mathrm{~g}^{-1} \mathrm{~K}^{-1}\right)$ & 3.74 & 4 & $8\left(5 \times 10^{-9} \times T^{3}-3 \times 10^{-6} \times T^{2}\right.$ & $8\left(4 \times 10^{-9} \times T^{3}-3 \times 10^{-6} \times T^{2}\right.$ \\
& & & $\left.+6 \times 10^{-4} \times T+0.31\right)$ & $\left.+8 \times 10^{-4} \times T+0.3\right)$
\end{tabular}


For all materials studied in this investigation, we estimate the absolute value of specific heat, $C_{E}(T, E)$ by adding the computed zero-field values of the excess specific heat $\Delta C_{E}(T, E)$ from eqn (3) to the lattice or hard-mode contributions taken from the experimental values.

\subsection{Experimental details}

This study has benefited from a substantial series of test data from prior experimental results, detailed extensively in ref. 25 and 37-41. The (total) heat capacity at constant electric field $E\left(C_{E}\right)$ was determined using either a high-resolution calorimeter described in ref. 25, 38 and 41, or a conventional Quantum Design Physical Properties Measurement System (PPMS) technique for all materials analyzed in this study except for PT. There is a quantitative and qualitative agreement between the calculated and measured heat capacities as discussed in ref. 37 . The experimental values of $C_{E}$, which consists of the excess heat capacity due to the spontaneous polarization as given in eqn (3) and lattice (hard mode) contributions, allow us to evaluate the theoretical adiabatic temperature changes $\left(\Delta T_{\mathrm{EC}}\right)$ with the application of an electric field difference $\Delta E$ [see eqn (5)].

In addition, there exists experimental data on $\Delta T_{\mathrm{EC}}$ for $0.90 \mathrm{PMN}-0.10 \mathrm{PT}$ and $0.65 \mathrm{PMN}-0.35 \mathrm{PT}$ which were measured directly using the methods given in ref. 2 and 37-40. These experimental results provide us an opportunity to compare theoretical and experimental work, at least in the low electric field regime. The details of our previous work on 0.90PMN$0.10 \mathrm{PT}$ and $0.65 \mathrm{PMN}-0.35 \mathrm{PT}$ can be found in ref. 39. Briefly, the materials in that study were polished single crystals that are perpendicularly cut to the [110]. For direct EC measurements, a high-resolution calorimeter with small modifications to the experimental setup has been utilized to measure a sample temperature variation due to the EC when an external field was applied. A step voltage form is applied to all the samples by a high-voltage generator. The sample temperature was acquired by a small bead thermistor attached to the sample electrode. The surrounding bath temperature was stabilized by a temperature controller within $0.1 \mathrm{mK}$. In typical sequence the field was switched on to some level for 500 seconds and then switched off. During this time and additional 500 seconds after the switching off the field the relaxation of the sample's temperature was monitored. This allows detailed analysis of the amplitude of the EC temperature change induced by the electric field as described in details in ref. 2.

\section{Analysis of results}

\subsection{Polarization, heat capacity, and pyroelectric coefficient}

In Fig. 1, we plot the equilibrium (spontaneous) polarization $P^{0}$ as functions of $T$ and applied electric field $E$ for PT, BT, 0.90PMN-0.10PT, and 0.65PMN-0.35PT. As expected, BT and PT display first-order phase transitions as described by the jump in $P^{0}$ for $E=0$ at the $\mathrm{FE}-\mathrm{PE}$ transformation temperature $T_{\mathrm{C}}$. In $\mathrm{BT}$, this first-order phase transition is weaker compared

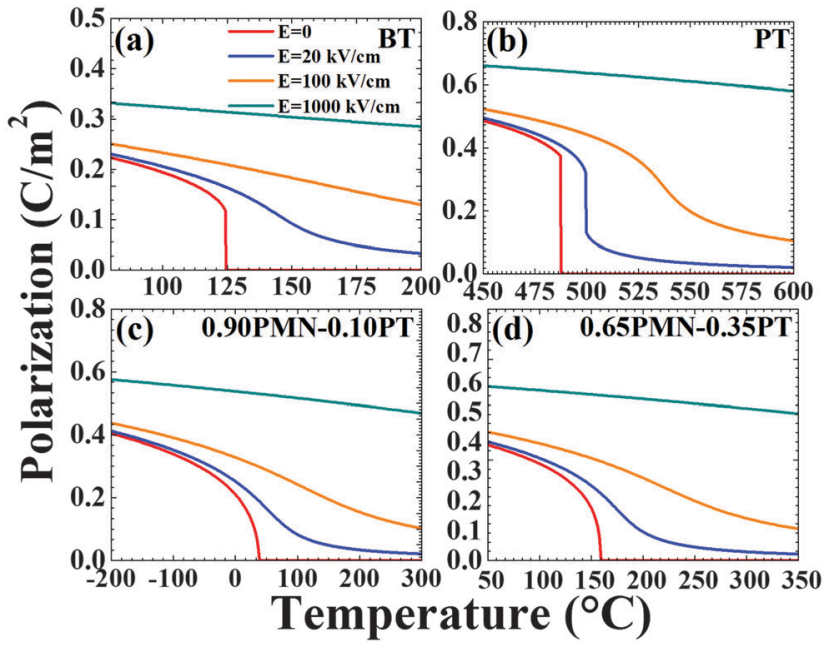

Fig. 1 Theoretical polarization variations: temperature and electric field dependent polarization variations of (a) BT, (b) PT, (c) 0.90PMN-0.10PT, and (d) 0.65PMN-0.35PT (labels for (b), (c), and (d) are the same as (a)).

to PT as characterized by the magnitude of the polarization jump at the respective $T_{\mathrm{C}} \mathrm{s}$. An applied electric field in the direction of the spontaneous polarization "smears" the phase transformation and it destroys the discontinuities in thermodynamic properties that are a function of polarization at the phase transition temperature. For BT, a field as high as $20 \mathrm{kV} \mathrm{cm}^{-1}$ is sufficient to wash out the first-order phase transition where as in PT, higher fields are required $\left(E=100 \mathrm{kV} \mathrm{cm}^{-1}\right)$. On the other hand, both PMN-PT compositions display a secondorder phase transitions. Here, there is no discontinuity at $T_{\mathrm{C}}$ in the polarization response and the effect of the applied electric field is similar resulting in a smearing of the phase transformation.

In Fig. 2 and 3 we plot the temperature dependence of the $p$ and the $C_{E}^{\mathrm{XS}}$ for the four materials investigated for $E=0,20,100$, and $1000 \mathrm{kV} \mathrm{cm}^{-1}$. These parameters are defined through the

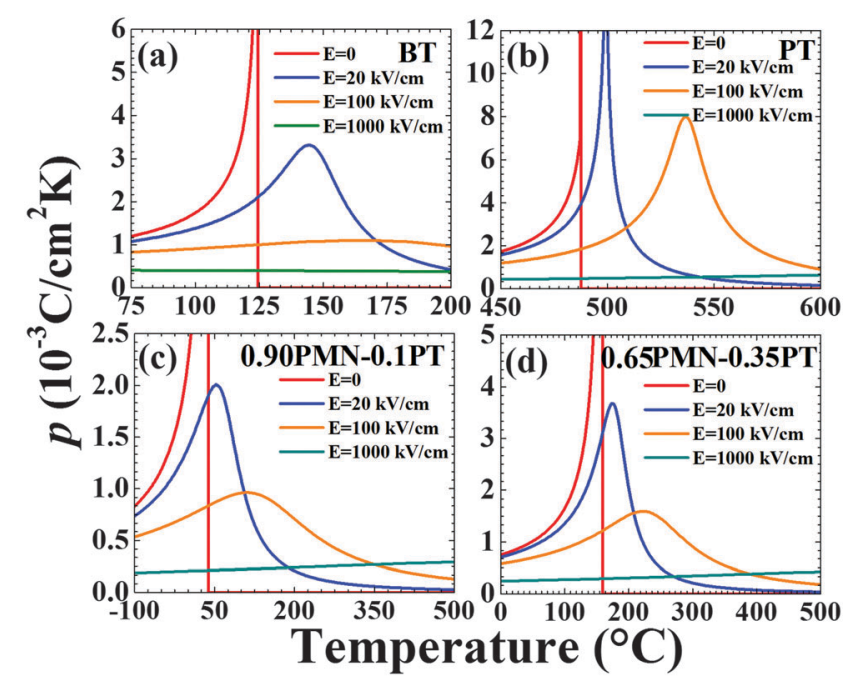

Fig. 2 Theoretical pyroelectric behavior: pyroelectric coefficient, $p$, of (a) $\mathrm{BT}$, (b) PT, (c) 0.90PMN-0.10PT, and (d) 0.65PMN-0.35PT as a function of temperature and applied electric field $E$. 


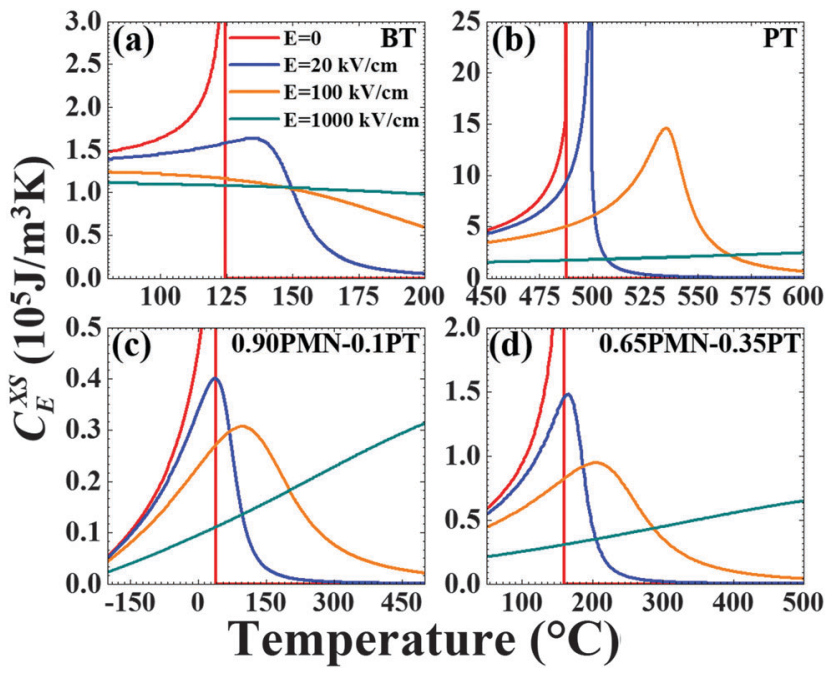

Fig. 3 Theoretical heat capacity variations: excess heat capacity of (a) BT, (b) PT, (c) 0.90PMN-0.10PT, and (d) 0.65PMN-0.35PT as a function of temperature and applied electric field $E$ (labels for (b), (c), and (d) are the same as (a)).

Maxwell equations as described under "Materials and Methods" and are essentially partial derivatives of the polarization with respect to temperature. As such, near $T_{\mathrm{C}}$, these quantities display a $\lambda$-type anomaly and this anomaly disappears with the application of an electric field in the direction of the equilibrium polarization. The behavior shown in Fig. 1-3 are expected responses and the relevant thermodynamic background can be found elsewhere. ${ }^{36}$

\subsection{Adiabatic temperature change}

It is necessary to experimentally determine or to be able to compute the reversible $\Delta T_{\mathrm{EC}}$ near and away from the $T_{\mathrm{C}}$ as this is a critical parameter in the estimation of the system-level coefficients of performance. The temperature dependence of $\Delta T_{\mathrm{EC}}$ as functions of $T$ and $\Delta E$ are thus plotted in Fig. 4. For all four materials, the field change $\Delta E$ is taken relative to an initial bias of $E_{a}=50 \mathrm{kV} \mathrm{cm}{ }^{-1}$. Fig. $4 \mathrm{a}$ plots $\Delta T_{\mathrm{EC}}$ as a function of applied field difference and temperature for BT. Near $T_{\mathrm{C}}$ $\left(121{ }^{\circ} \mathrm{C}\right), \Delta T_{\mathrm{EC}}$ is computed to be $0.8,2.8,4.4$, and $7.3{ }^{\circ} \mathrm{C}$ for $\Delta E=50,250,500$, and $1000 \mathrm{kV} \mathrm{cm}^{-1}$. The experimental data points are taken from ref. 37 where $\Delta T_{\mathrm{EC}}$ is obtained through direct high-resolution EC measurements for BT single-crystals oriented along the [001]. Although the applied fields are different and the initial fields are relatively small, $\Delta T_{\mathrm{EC}}$ is larger compared to theoretical predictions $\left(1.5{ }^{\circ} \mathrm{C}\right.$ for $\left.\Delta E=28 \mathrm{kV} \mathrm{cm}^{-1}\right)$. We attribute this difference both due to extrinsic contributions to the EC effect, and the fact that we do not account for the nonzero entropy change between zero applied field and our lower bound value.

For both $0.90 \mathrm{PMN}-0.10 \mathrm{PT}$ and $0.90 \mathrm{PMN}-0.35 \mathrm{PT}$, the $\Delta T_{\mathrm{EC}}$ responses are much broader and relatively less temperature sensitive as shown in Fig. $4 \mathrm{c}$ and d, respectively, compared to $\mathrm{BT}$ and PT. In the case of 0.90PMN-0.10PT, $\Delta T_{\mathrm{EC}}=0.8,2.9,4.9$, and $8.8{ }^{\circ} \mathrm{C}$ for $\Delta E=50,250,500,1000 \mathrm{kV} \mathrm{cm}^{-1}$, respectively,

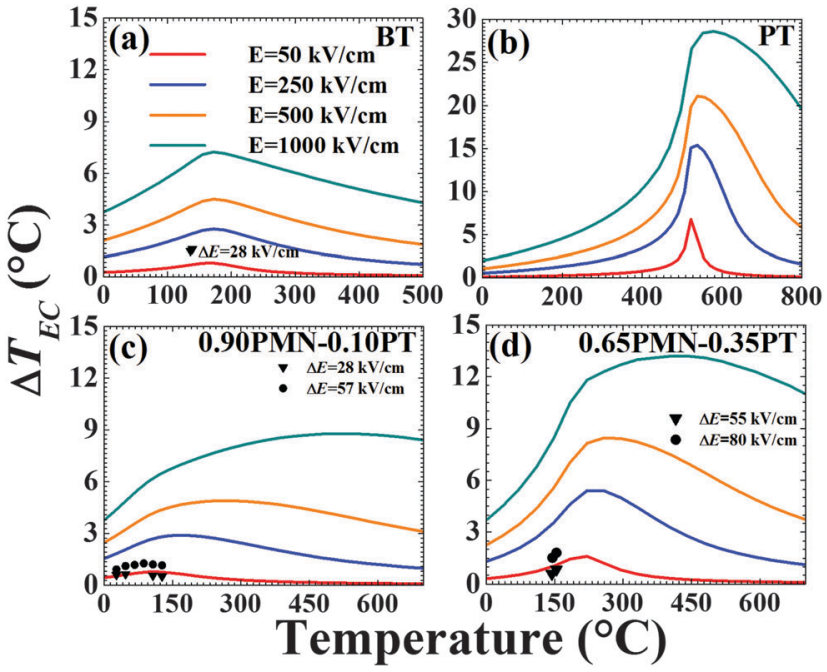

Fig. 4 Theoretical adiabatic temperature changes: EC adiabatic temperature change, $\Delta T_{\mathrm{EC}}$, of (a) $\mathrm{BT}$, (b) $\mathrm{PT}$, (c) $0.90 \mathrm{PMN}-0.10 \mathrm{PT}$, and (d) $0.65 \mathrm{PMN}-0.35 \mathrm{PT}$ as a function of temperature for $\Delta E$ ranging from 50 to $1000 \mathrm{kV} \mathrm{cm}{ }^{-1}$. The initial (biasing field) $E_{a}$ is taken as $50 \mathrm{kV} \mathrm{cm}^{-1}$. Also shown are experimentally measured EC data points (solid symbols) in $\mathrm{BT}, 0.90 \mathrm{PMN}-0.10 \mathrm{PT}$, and 0.65PMN-0.35PT samples $(2,33,43)$.

near its $T_{\mathrm{C}}$ of $38{ }^{\circ} \mathrm{C}$. For $0.65 \mathrm{PMN}-0.35 \mathrm{PT}\left(T_{\mathrm{C}}=159{ }^{\circ} \mathrm{C}\right)$, the EC response is considerably larger; $\Delta T_{\mathrm{EC}}=1.6,5.5,8.5$, and $13.1{ }^{\circ} \mathrm{C}$ for $\Delta E=50,250,500,1000 \mathrm{kV} \mathrm{cm}^{-1}$, respectively. In the two PMN-PT systems (Fig. 4c and d), we found good agreement with directly measured EC data at lower fields (solid symbols). This difference can again be related to extrinsic contributions. Furthermore, experimentally, we start from zero field thus invoking the latent heat release (for fields below $14 \mathrm{kV} \mathrm{cm}^{-1}$ which is critical field to induce the liquid-vapor type critical point in this system above which there is no contribution from a latent heat ${ }^{39}$ ), which in turn enhances the EC effect in contrast to our calculation in which field change starts from $50 \mathrm{kV} \mathrm{cm}^{-1}$. Thus, because essentially no latent heat is released and we probe only continuous variations of the total entropy $(S)$ above the discontinuous jump in $S$ our theoretical results are diminished compared to experiment.

For PT, the EC response near $T_{\mathrm{C}}=490{ }^{\circ} \mathrm{C}$ is significantly larger, where $\Delta T_{\mathrm{EC}}=3.2,15.0,21.1$, and $28.5{ }^{\circ} \mathrm{C}$ for $\Delta E=$ $50,250,500$, and $1000 \mathrm{kV} \mathrm{cm}^{-1}$ (Fig. 4b). We attribute this behavior to the more pronounced first-order phase transformation in PT compared to BT, as discussed in detail by Zhang et $a l .{ }^{42}$ The "jump" in the polarization (and hence the entropy change) at $T_{\mathrm{C}}$ in PT is associated with the relatively large displacement of the $\mathrm{Ti}^{4+}$ ions with respect to the $\mathrm{O}^{2-}$ ions. In PT single crystals the eigenstrain at $\mathrm{RT}$ is as large as $3.67 \% .{ }^{43}$ The large intrinsic non-centrosymmetric displacement below $T_{\mathrm{C}}$ corresponds to a large entropy variation when compared to the non-polarized centrosymmetric PE phase. This displacement is considerably larger in PT compared to the BT and 0.90PMN-0.10PT and 0.65PMN$0.35 \mathrm{PT}\left(0.70 \%, 0.08 \%\right.$, and $1.30 \%$, respectively, at $\left.\mathrm{RT}^{44-46}\right)$ and this is the reason for the significantly larger $\Delta T_{\mathrm{EC}}$ near their respective $T_{\mathrm{C}} \mathrm{S}$. 


\section{Discussion}

For $E=0$, there is a $\lambda$-anomaly associated with the first-order FE to $\mathrm{PE}$ phase transformation, resulting in (theoretically) an infinitely large $p$ and $C_{E}^{\mathrm{XS}}$ at the equilibrium transition temperature $T_{\mathrm{C}}$. The application of high enough $E$ along the polarization direction results in the smearing of the temperature dependence of $p$ and $C_{E}^{\mathrm{XS}}$ as shown in Fig. 2 and 3 for all four materials. This results in the disappearance of welldefined maxima in these properties at sufficiently large electric fields: the maxima in $p$ and $C_{E}^{\mathrm{xs}}$ shift to higher temperatures and are reduced in magnitude with an increase in $E$. Furthermore, these quantities become progressively less sensitive to temperature for larger fields. In the case of PT, however, owing to a much stronger first-order FE-PE phase change as defined by the jump in the spontaneous polarization at $T_{\mathrm{C}}$ (see Fig. 1), relatively higher electric fields are required to achieve the same effect. At lower field difference, all four materials show typical FE behavior such that there is an increase in the value $\Delta T_{\mathrm{EC}}$ with operating temperature, up to a point around their respective $T_{\mathrm{C}}$. There is a decrease in $\Delta T_{\mathrm{EC}}$ beyond a maximum near the $T_{\mathrm{C}}$ with increasing temperature which is expected ${ }^{42}$ since the pyroelectric response for $T>T_{\mathrm{C}}$ for small electric fields is essentially given by the variation of the electric field induced polarization in the PE phase. For significantly higher fields, the EC response of PT and BT shows a maximum in $\Delta T_{\mathrm{EC}}$ near $T_{\mathrm{C}}$ and a decrease for $T>T_{\mathrm{C}}$. However, in the cases of $0.90 \mathrm{PMN}-$ $0.10 \mathrm{PT}$ and $0.65 \mathrm{PMN}-0.35 \mathrm{PT}, \Delta E=1000 \mathrm{kV} \mathrm{cm}^{-1}$ is sufficiently large to obscure the effect of the phase transition at $T_{\mathrm{C}}$, at least for temperatures in which the material retains it ferroelectric properties. Such a difference in behavior can be assigned, at least in part, to the presence of a relaxor nature of the FE solid solutions, and the accompanying dispersion of the FE-PE transition. ${ }^{47}$ Application of a large enough electric field can also be expected to result in a partial stabilization of the FE phase to slightly higher temperatures than $T_{\mathrm{C}} \cdot{ }^{48}$ Also, as it can be seen in Fig. 5, both 0.90PMN-0.10PT and 0.65PMN-0.35PT have a relatively temperature insensitive $\Delta T_{\mathrm{EC}}$ over a larger temperature range compared to $\mathrm{PT}$ and $\mathrm{BT}$. The large peak in EC response of the PMN-PT composition corresponding to the MPB is similar to the previously calculated (and experimentally confirmed) peaks in the dielectric, piezoelectric and elastic compliance at the MPB for lead zirconate-lead titanate (PZT) solid solutions. ${ }^{36}$ The enhancement in the properties are often attributed to the co-presence of two (or more) phases with differing polarization directions. This could be related to the disappearance of anisotropy contributions to the free energy and formation of nano-domains. ${ }^{36,49}$

We note that the calculated values in this work are limited to intrinsic EC response. Effective functional responses in ferroic materials are due to both their intrinsic (lattice) and extrinsic nature which includes motion of domain walls, formation of phase boundaries, and local (defect) dipoles. While the amount of extrinsic contributions to the effective EC response has not been quantified, such data are indeed available for the dielectric response, and in part, also for the piezoelectric response of the

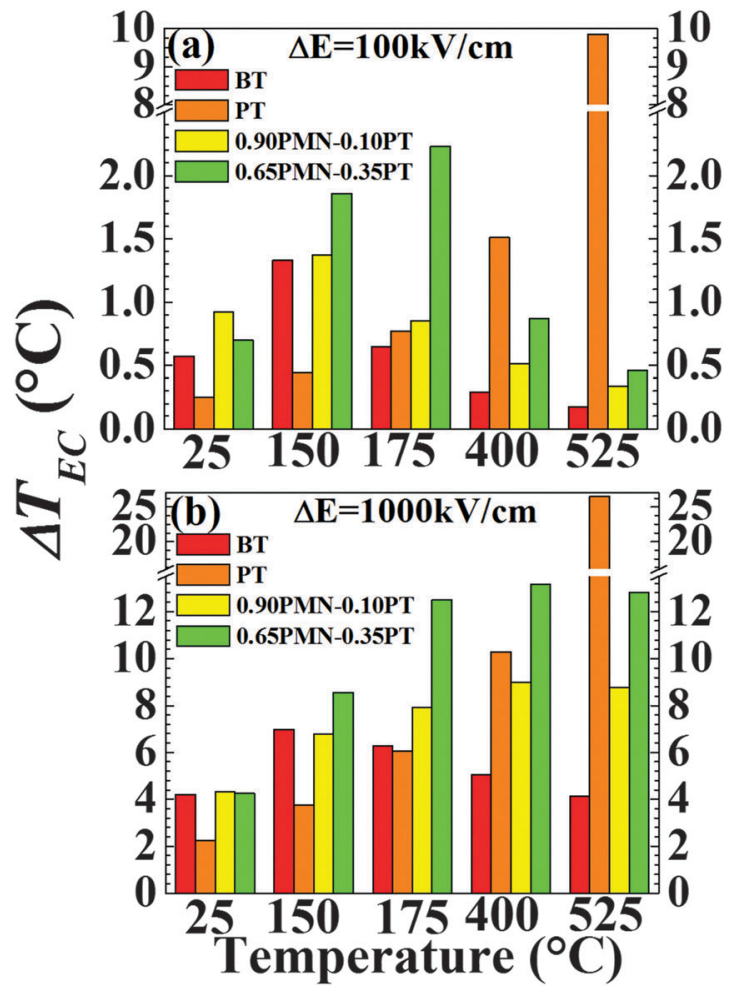

Fig. 5 Theoretical adiabatic temperature change: comparative adiabatic temperature change, $\Delta T_{\mathrm{EC}}$, of $\mathrm{BT}, \mathrm{PT}, 0.90 \mathrm{PMN}-0.10 \mathrm{PT}$, and 0.65PMN$0.35 \mathrm{PT}$ at different working temperatures: (a) $\Delta E=100 \mathrm{kV} \mathrm{cm}^{-1}$ and (b) $\Delta E=1000 \mathrm{kV} \mathrm{cm}^{-1}$. The initial (biasing field) $E_{a}$ is taken as $50 \mathrm{kV} \mathrm{cm}^{-1}$.

materials considered here. In general, relaxor-FEs such as PMN-PT have been shown to exhibit enhanced extrinsic properties that can be as high as $30-80 \%$ of the total functional response in BT and PZT ceramics. ${ }^{50}$ This contribution could be significantly larger in relaxor- $\mathrm{PbTiO}_{3}$ near MPB single crystals, evaluated to be $\sim 300 \%$. ${ }^{51}$ We can, therefore, expect that the overall EC response of these single crystals to be similarly augmented by the extrinsic contributions, and through domain wall engineering, i.e. creation of specific mobile (adaptive) domain architectures to further enhance entropy variations with an applied electric field. This enhancement should be more pronounced at relatively low initial biases. Finally, we show in Fig. 5 a more detailed comparison of the EC response of all four materials at five specific temperatures $(T=25,150,175,400$ and $525{ }^{\circ} \mathrm{C}$ ) at an intermediate field of $\Delta E=100 \mathrm{kV} \mathrm{cm}^{-1}$ (Fig. 5a) and a relatively higher field of $\Delta E=1000 \mathrm{kV} \mathrm{cm}^{-1}$ (Fig. 5b). In both cases, $E_{a}$ was taken to be $50 \mathrm{kV} \mathrm{cm}^{-1}$.

\section{Conclusions}

In summary, we have calculated the EC temperature changes in BT, PT, 0.90PMN-0.10PT, and 0.65PMN-0.35PT over a wide range of temperatures. Large EC responses close to or exceeding $10{ }^{\circ} \mathrm{C}$ were found over a very broad temperature range exceeding several hundred degrees in case of BT, and two PMN-PT systems. In PT, a giant EC response of $28{ }^{\circ} \mathrm{C}$ at $1 \mathrm{MV} \mathrm{cm}^{-1}$ is predicted. 
These adiabatic temperature changes correspond to significant entropy variations. For example, $\Delta S=-22.16$ and $-7.29 \mathrm{~J} \mathrm{~kg}^{-1} \mathrm{~K}^{-1}$ for PT and 0.65PMN-0.35PT, respectively for temperatures at which each material shows the maximum $\Delta T_{\mathrm{EC}}\left(561{ }^{\circ} \mathrm{C}\right.$ for $\mathrm{PT}$ and $253{ }^{\circ} \mathrm{C}$ for $0.65 \mathrm{PMN}-0.35 \mathrm{PT}$ ). This is similar to some of the best EC responses experimentally observed at similar fields in polymeric FEs. It should be noted that $\Delta T_{\mathrm{EC}}(\Delta E)$ shows little saturation at large fields indicating that in these materials truly very large EC response is achievable. ${ }^{52}$ Therefore, it may prove optimal for application to operate these materials at large fields as demonstrated recently in thick films. Lastly, we note that many of the experimental studies of ceramic based materials have been performed on EC films clamped to a substantially thicker substrate. Considering that this two-dimensional clamping reduces all property coefficients including the pyroelectric and EC response, ${ }^{42,53}$ our analysis provides a guide to the experimentalist and application community, in as much as it predicts that the highest EC responses will be best achieved by removing the constraints limiting the full performance of these materials.

\section{Acknowledgements}

Z. K. acknowledges support by the Slovenian Research Agency through Grant No. P1-0125 and J2-6779. N. B. G. gratefully acknowledges funding from the US National Science Foundation through grants DMR-1255379 and CMMI-1537262.

\section{References}

1 A. S. Mischenko, Q. Zhang, J. F. Scott, R. W. Whatmore and N. D. Mathur, Science, 2006, 311, 1270-1271.

2 Z. Kutnjak, B. Rožič and R. Pirc, Wiley Encyclopedia of Electrical and Electronics Engineering, 2015.

3 I. Takeuchi and K. Sandeman, Phys. Today, 2015, 68, 48.

4 S. P. Alpay, J. M. Mantese, S. Trolier-McKinstry, Q. Zhang and R. W. Whatmore, MRS Bull., 2014, 39, 1099-1111.

5 T. M. Correia, S. Kar-Narayan, J. S. Young, J. F. Scott, N. D. Mathur, R. W. Whatmore and Q. Zhang, J. Phys. D: Appl. Phys., 2011, 44, 165407.

6 S. G. Lu and Q. Zhang, Adv. Mater., 2009, 21, 1983-1987.

7 M. Valant, Prog. Mater. Sci., 2012, 57, 980-1009.

8 J. F. Scott, Annu. Rev. Mater. Res., 2011, 41, 229-240.

9 M. Ožbolt, A. Kitanovski, J. Tušek and A. Poredoš, Int. J. Refrig., 2014, 40, 174-188.

10 F.-C. Sun, A. M. Dongare, A. D. Asandei, S. P. Alpay and S. Nakhmanson, J. Mater. Chem. C, 2015, 3(32), 8389-8396.

11 V. F. Freitas, O. A. Protzek, L. A. Montoro, A. M. Gonçalves, D. Garcia, J. A. Eiras, R. Guo, A. S. Bhalla, L. F. Cótica and I. A. Santos, J. Mater. Chem. C, 2014, 2(2), 364-372.

12 J. Varghese, R. W. Whatmore and J. D. Holmes, J. Mater. Chem. C, 2013, 1(15), 2618-2638.

13 N. Bassiri-Gharb, Y. Bastani and A. Bernal, Chem. Soc. Rev., 2014, 43(7), 2125-2140.
14 Q. Li, G. Zhang, X. Zhang, S. Jiang, Y. Zeng and Q. Wang, Adv. Mater., 2015, 27, 2236-2241.

15 Y. Liu, I. C. Infante, X. Lou, L. Bellaiche, J. F. Scott and B. Dkhil, Adv. Mater., 2014, 26, 6132-6137.

16 R. Ramesh, S. Aggarwal and O. Auciello, Mater. Sci. Eng., R, 2001, 32, 191-236.

17 P. Yudin and A. Tagantsev, Nanotechnology, 2013, 24, 432001.

18 H. Khassaf, N. Khakpash, F. Sun, N. M. Sbrockey, G. S. Tompa, T. S. Kalkur and S. P. Alpay, Appl. Phys. Lett., 2014, 104, 202902.

19 F. Jin, G. W. Auner, R. Naik, N. W. Schubring, J. V. Mantese, A. B. Catalan and A. L. Micheli, Appl. Phys. Lett., 1998, 73, 2838-2840.

20 I. A. Kornev, L. Bellaiche, P.-E. Janolin, B. Dkhil and E. Suard, Phys. Rev. Lett., 2006, 97, 157601.

21 J. Lutsko, D. Wolf, S. Yip, S. Phillpot and T. Nguyen, Phys. Rev. B: Condens. Matter Mater. Phys., 1988, 38, 11572.

22 S. R. Phillpot, S. B. Sinnott and A. Asthagiri, Annu. Rev. Mater. Res., 2007, 37, 239-270.

23 N. Khakpash, H. Khassaf, G. A. Rossetti Jr and S. P. Alpay, Appl. Phys. Lett., 2015, 106, 082905.

24 K. Bhattacharya and G. Ravichandran, Acta Mater., 2003, 51, 5941-5960.

25 N. Novak, G. Cordoyiannis and Z. Kutnjak, Ferroelectrics, 2012, 428, 43-48.

26 A. Mischenko, Q. Zhang, R. W. Whatmore and N. Mathur, Appl. Phys. Lett., 2006, 89, 242912.

27 Y. Zhang, G. Gao, H. L. Chan, J. Dai, Y. Wang and J. Hao, Adv. Mater., 2012, 24, 1729-1735.

28 L. Bellaiche and D. Vanderbilt, Phys. Rev. Lett., 1999, 83, 1347.

29 Y. Sato, T. Hirayama and Y. Ikuhara, Appl. Phys. Lett., 2012, 100, 172902.

30 V. Vikhnin, R. Blinc and R. Pirc, J. Appl. Phys., 2003, 93, 9947-9952.

31 S. G. Lu, B. Rozic, Q. M. Zhang, Z. Kutnjak, R. Pirc, M. Lin and L. Gorny, Appl. Phys. Lett., 2010, 97, 202901.

32 G. Zhang, Q. Li, H. Gu, S. Jiang, K. Han, M. R. Gadinski, M. A. Haque, Q. Zhang and Q. Wang, Adv. Mater., 2015, 27, 1450-1454.

33 I. Ponomareva and S. Lisenkov, Phys. Rev. Lett., 2012, 108, 167604.

34 R. Pirc, Z. Kutnjak, R. Blinc and Q. Zhang, Appl. Phys. Lett., 2011, 98, 1909.

35 A. A. Heitmann and G. A. Rossetti, J. Am. Ceram. Soc., 2014, 97, 1661-1685.

36 G. Akcay, S. P. Alpay, G. A. Rossetti Jr and J. F. Scott, J. Appl. Phys., 2008, 103, 024104.

37 N. Novak, Z. Kutnjak and R. Pirc, EPL, 2013, 103, 47001.

38 Z. Kutnjak, J. Petzelt and R. Blinc, Nature, 2006, 441, 956-959.

39 N. Novak, R. Pirc, M. Wencka and Z. Kutnjak, Phys. Rev. Lett., 2012, 109, 037601.

40 G. Sebald, L. Seveyrat, D. Guyomar, L. Lebrun, B. Guiffard and S. Pruvost, J. Appl. Phys., 2006, 100, 124112.

41 D. Guyomar, G. Sebald, B. Guiffard and L. Seveyrat, J. Phys. D: Appl. Phys., 2006, 39, 4491. 
42 J. Zhang, A. A. Heitmann, S. P. Alpay and G. A. Rossetti Jr, J. Mater. Sci., 2009, 44, 5263-5273.

43 G. Shirane, H. Sadao and S. Kazuo, Phys. Rev., 1950, 80(6), 1105.

44 M. H. Frey and D. A. Payne, Phys. Rev. B: Condens. Matter Mater. Phys., 1996, 54(5), 3158.

45 J. C. Ho, K. S. Liu and I. N. Lin, J. Mater. Sci., 1993, 28(16), 4497-4502.

46 Z.-G. Ye, Y. Bing, J. Gao, A. A. Bokov, P. Stephens, B. Noheda and G. Shirane, Phys. Rev. B: Condens. Matter Mater. Phys., 2003, 67(10), 104104.

47 A. A. Bokov and Z.-G. Ye, J. Adv. Dielectr., 2012, 2, 1241010.
48 L. Garten, P. Lam, D. Harris, J.-P. Maria and S. TrolierMcKinstry, J. Appl. Phys., 2014, 116, 044104.

49 D. Damjanovic, Appl. Phys. Lett., 2010, 97, 062906.

50 D. Damjanovic and M. Demartin, J. Phys.: Condens. Matter, 1997, 9, 4943.

51 D. Lin, S. Zhang, Z. Li, F. Li, Z. Xu, S. Wada, J. Luo and T. R. Shrout, J. Appl. Phys., 2011, 110, 084110.

52 S. Lu, B. Rožič, Q. Zhang, Z. Kutnjak, X. Li, E. Furman, L. J. Gorny, M. Lin, B. Malič and M. Kosec, Appl. Phys. Lett., 2010, 97, 162904.

53 J. Zhang, M. W. Cole and S. P. Alpay, J. Appl. Phys., 2010, 108(5), 054103. 